\title{
Numerical Calculation and Design of Variant Topologies of Printed Dipole Antennas
}

\author{
Clément Mbinack ${ }^{1}$ Emmanuel Tonye ${ }^{2}$ \\ ${ }^{1}$ Laboratory of Energy and Electrical and Electronic Systems, Department of Physic, Faculty of Sciences, University of Yaoundé I, \\ P.O Box 812 Yaoundé-Cameroon \\ ${ }^{2}$ Laboratory of Electronics and Signal Processing, National Advanced School of Engineering, University of Yaoundé I, P.O Box 8390 \\ Yaoundé-Cameroon
}

\begin{abstract}
We define the gain of an antenna through reference to a standard antenna. The reference antenna is amongst other things a perfect antenna which radiates in the same manner in all space directions with a circular radiation pattern. In reality this isotropic antenna does not exist; this is an imaginary antenna; we do not know how to achieve it in practice; but it is convenient to serve as a standard to test the real antennas. We therefore propose in this paper to design a model which is very close to the perfect antenna achieved from the popular microstrip dipole antenna working at frequency range of $2.4 \mathrm{GHz}$.
\end{abstract}

Keywords: coplanar waveguide, mono-plane microstrip dipole antenna, radiation pattern, input impedance.

\section{Introduction}

Printed dipole antennas are not vastly in use and their employment remains limited to a few applications due to the fact that they do not radiate into their own direction [1]. However, microstrip dipole antennas appear today as prospective elements because of their low weight, of their excellent RF performance and of their ease of fabrication [2]. Currently efforts are being made and are oriented towards on innovation and the exploration of new topologies of printed dipole antennas using numerical simulations [3]-[5].

The approach envisaged by most researchers to achieve this new requirement press hold on the evaluation of the performances of new developed models using electromagnetic simulations and experimental measurements [6], [7]. Using a simplified integral equation and solved in the frequency domain by the way of the method of moments, Laohapensaeng in [8] studied the behavior of the input impedance of the microstrip dipole antenna. This experimental study takes into account the effects of the dielectric substrate on the electrical characteristics of the antenna and enhances its bandwidth. In [9] the impact of the bend on microstrip line and the dipole's gap on reflection coefficient and radiation pattern of printed dipole antenna has been investigated. To analyze the radiation characteristics, some numerical and calculation methods are proposed in [10]. In [2] a compact printed dipole antenna with wide impedance bandwidth is studied. Jamaluddin in [6] has investigated the effects of the geometry of planar dipole on its input impedance and proposed an impedance-matching method based on the antenna structure, which allows the enhancement of the bandwidth.

In this work, we try to explore the potentialities of new topologies of printed dipole antennas in a coplanar configuration. Topologies involved here are obtained by changing the profile or the physical form of the known printed dipole architecture. The corresponding printed dipole antennas obtained are I, L, Z, half ring and other more complex forms. A first approach to investigate the proposed structure is based on the Calculation of radiation patterns. In the second approach we presented design principle and considerations based on numerical simulations that allow the evaluation of the input impedance of the studied antennas. Calculation and Simulated results compared with experimental measurements clearly show a good agreement with theoretical predictions.

\section{Radiation Pattern of Mono-Plane Printed Dipole Antenna Calculation}

Mono-plane microstrip dipole antenna in I-topology consists of a pair of radiation metal arms of width $W_{0}$ and of total length $l=\lambda_{g} / 2$ printed on one side of a dielectric substrate of permittivity $\varepsilon$ (respectively of permeability $\mu$ ) and thickness $h$. The other side of the substrate is without any metallization. It is fed at its center by a coaxial probe as illustrated in figure 1.

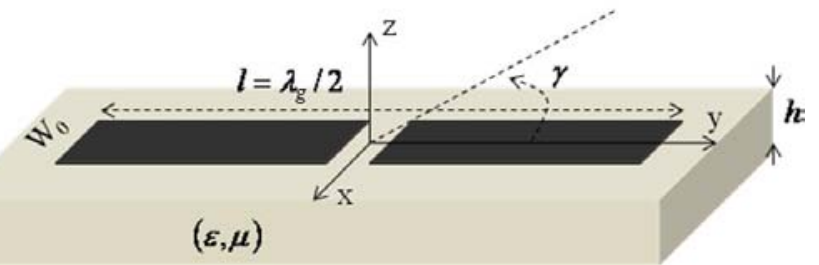

Figure 1: Probe-fed printed mono-plane dipole antenna in Itopology

Given the low values firstly of the substrate thickness ( $h<<W_{0}$ ) and on the other hand of the width of the radiating strands $\left(W_{0}<<\lambda_{\text {eff }}\right)$, all can be likened to the wire dipole with a sinusoidal axial current of the form:

$$
\begin{array}{r}
J(y)=J_{0} \sin \left[k_{\text {eff }}(l-|y|)\right] \\
\text { where: } J_{0} \cong \frac{2}{\pi W_{0}} ; k_{\text {eff }}=\frac{k_{0}}{\sqrt{\varepsilon_{\text {eff }}}} ;
\end{array}
$$




\section{International Journal of Science and Research (IJSR) \\ ISSN (Online): 2319-7064}

Index Copernicus Value (2013): 6.14 $\mid$ Impact Factor (2015): 6.391

$$
\varepsilon_{e f f}=1+\frac{\varepsilon_{r}-1}{2}\left[1-\left(\frac{W_{0} / h}{1+W_{0} / h}\right)\right] \text { and } \lambda_{\text {eff }}=\frac{\lambda_{0}}{\sqrt{\varepsilon_{e f f}}}
$$

The $\theta$-component of the electric field radiated in Fraunhofer (or far) zone in $\gamma$-direction that the antenna is made with the radiation direction (see fig. 1 ) is given by:

$$
E_{\theta}(\theta, \phi)=-j \omega \frac{\mu_{0} J_{0}}{4 \pi} f(\theta, \phi) g(\theta, \phi)
$$

where:

$$
\begin{aligned}
& \left\{\begin{array}{l}
f(\theta, \phi)=\cos (\theta) \cos (\phi) \\
g(\theta, \phi)=\iint_{s} \sin \left[k_{e f f}(l-|y|)\right] e^{j\left(k_{x} x+k_{y} y\right)} d x d y
\end{array}\right. \\
& -\frac{W_{0}}{2}<x<+\frac{W_{0}}{2} ;-\frac{\lambda_{g}}{4}<y<+\frac{\lambda_{g}}{2} ; \cos (\gamma)=\sin (\theta) \sin (\phi)
\end{aligned}
$$

From this expression, we can deduce the pattern of any other printed dipole antenna made from the popular antenna shown in fig. 1 whatever its form.

\section{Design considerations of mono-plane microstrip dipole antenna in $L$ and $Z$ - topologies}

The explored new topologies of mono-plane microstrip dipole antennas illustrated in figure 2 take their origin in the research of an appropriate response to the raised problem of shadow regions during the led investigations on the radiation pattern produced by the printed dipole antenna in I-topology. As shown in figure 5, it should be noted that mono-plane microstrip dipole antenna in I-topology just like wire dipoles don't radiate in their own direction. To improve the radiation and the impedance-matching performances of mono-plane printed dipole antenna in I-topology, the bends at right angles are minutely made on the profile of this antenna.

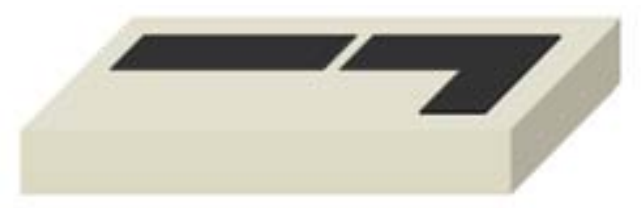

(a)

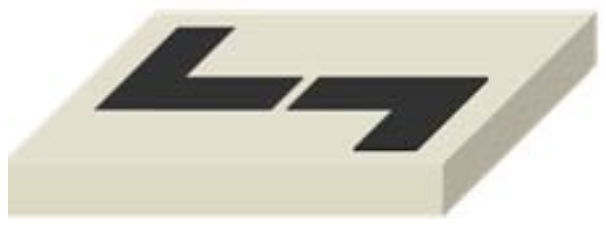

(b)

Figure 2: Probe-fed microstrip dipole antenna in (a) L-topology (b) Z-topology

Naturally, all of those thorough works of design and fabrication of new topologies of the proposed antennas must be conducted by maintaining constant the initial total length of the base antenna. The L-topology leads to an asymmetrical element while the Z-topology will keep the symmetry of the popular element in terms of geometry and current distribution. The very attractive idea of creating bends at right angle on the radiation metal arms placed in the Itopology generated a new current component perpendicular to the longitudinal current component of the known dipole antenna, which allows obtaining quasi-uniform radiation pattern. Other more complex topologies as shown in fig.3 can be explored with best performances in perspective.

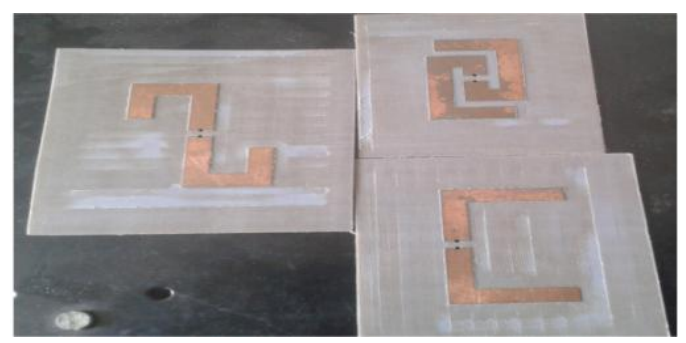

Figure 3: Other more complex structures which can further improve the antenna performances

\section{Input impedance calculation}

Mono-plane microstrip dipole antenna in I-topology can be modeled as a coplanar waveguide as depicted in fig. 4 .

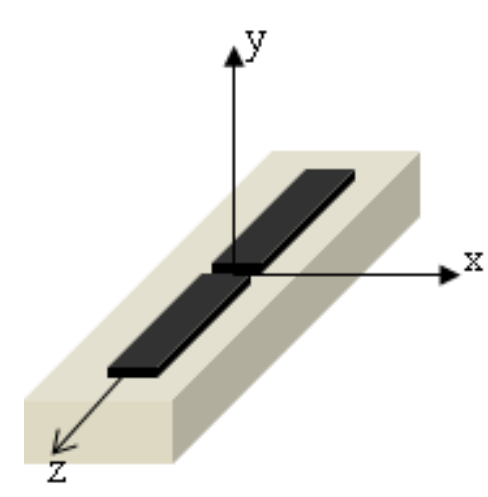

(a)

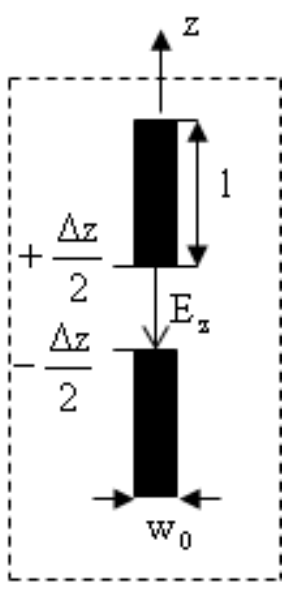

(b)
Figure 4: Printed dipole antenna in I-topology $\begin{array}{ll}\text { (a) coplanar waveguide model } & \text { (b) top view }\end{array}$ $\Delta z$ denote the excitation gap

For this electromagnetic model, fullwave analysis using Galerkin's method in spectral domain is usually employed. Hence in this work antenna is being treated in terms of field distribution within the waveguide to determine its input impedance.

For the boundary conditions it may be assume that at $z=+\frac{\Delta z}{2}, V\left(+\frac{\Delta z}{2}\right)=V_{0}$ and at $z=-\frac{\Delta z}{2}, V\left(-\frac{\Delta z}{2}\right)=-V_{0}$

In TM mode, the longitudinal component of electric field is given by the expression

$$
E_{z}=-j \frac{p^{2}}{k \eta} I(z) \Phi(x, y)
$$

where the potential $\Phi$ satisfied the propagation equation

$$
\nabla_{t} \Phi+p^{2} \Phi=0
$$

with $p^{2}=k^{2}-\beta^{2}$ and $k^{2}=\omega^{2} \mu \varepsilon$

For narrow substrate thickness, we assume that the dependence of the potential $\Phi$ into the $y$-coordinate is negligible and solving equation (4) then gives 


\section{International Journal of Science and Research (IJSR) \\ ISSN (Online): 2319-7064}

Index Copernicus Value (2013): 6.14 | Impact Factor (2015): 6.391

$$
\Phi(x) \propto \cos \left\lfloor\frac{m \pi}{W_{0 e f f}}\left(x-\frac{W_{0 e f f}}{2}\right)\right\rfloor
$$

For the lossless transmission line, we can write

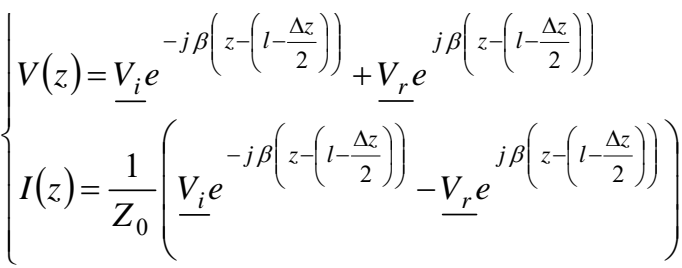

so that the general expression of the longitudinal electric field component become

$$
\begin{aligned}
E_{z}(x, z)= & E_{0}\left\lfloor e^{-j \beta\left(z-\left(l-\frac{\Delta z}{2}\right)\right)}-\Gamma_{L} e^{j \beta\left(z-\left(l-\frac{\Delta z}{2}\right)\right)}\right\rfloor \times \\
& \cos \left\lfloor\frac{m \pi}{W_{0 e f f}}\left(x-\frac{W_{0 e f f}}{2}\right)\right\rfloor
\end{aligned}
$$

where $E_{0}=\frac{p^{2} \underline{V_{i}}}{j k \eta Z_{0}}$

The input impedance may be calculated using the voltagecurrent definition by the expression

$$
Z(z)=\frac{V(z)}{I(z)}
$$

for which we deduce

$$
Z_{\text {in }}=Z\left(-\frac{\Delta z}{2}\right)+Z\left(+\frac{\Delta z}{2}\right)
$$

To calculate the load coefficient $\Gamma_{L}$ we consider only the field on the excitation gap where the field is constant to write

$$
E\left(0, \frac{\Delta z}{2}\right)=E_{0}=\frac{2 V_{0}}{\Delta z}
$$

Using electric field-voltage definition

$$
\vec{E}=-\vec{\nabla} V
$$

we can write on the excitation gap

$$
\frac{E_{0}}{j \beta}\left(e^{-j \beta(-l+\Delta z)}+\Gamma_{L} e^{j \beta(-l+\Delta z)}\right)=V_{0}
$$

that yield

$$
\Gamma_{L}=\Gamma_{0} e^{j \beta(l-\Delta z)}-e^{j 2 \beta(l-\Delta z)}
$$

where $\Gamma_{0}=\frac{j \beta V_{0}}{E_{0}}$

with $m=0,2,4, \ldots$

In the practice, the fact of making bends on the radiating arms should be normally done by maintaining constant, the total length of the common antenna. However, we observe that the innovative idea to make bends on the radiation metal arms rather leads to the decrease of the electrical length of the resonator and consequently, a shift of the resonant frequency. An adjustment of the total length of the antenna is therefore required during the bending process to try to bring the resonant frequency around the work frequency.

\section{Experimental Results and Discussion}

In this work we have use a dielectric substrate of relative dielectric constant $\varepsilon_{r}=2.2$, of thickness $h=0.635 \mathrm{~mm}$ and of loss tangent $10^{-3}$. In this antenna design, Agilent's ADS software using Momentum simulation that appear very suitable for electromagnetic modeling of planar structures is employed, whereas the experimental measurements have been taken with a bi-curve network analyzer. Mono-plane microstrip dipole antenna in I, L and Z-topologies of total length $l=54 \mathrm{~mm}$ and width $W_{0}=4 \mathrm{~mm}$, have been investigated.

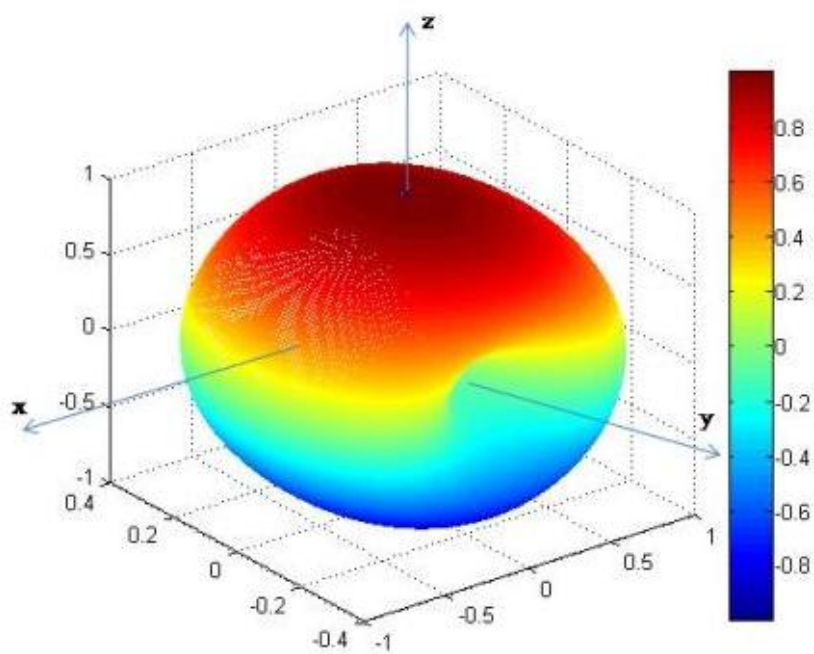

Figure 5: 3-D radiation pattern of microstrip dipole antenna Figure 5 gives 3-D radiation pattern of mono-plane microstrip dipole antenna in I-Topology. This radiation pattern is close enough to the radiation pattern of the Hertz's dipole. We observe well the existence of shadow regions in the antenna direction parallel to the $y$-axis.

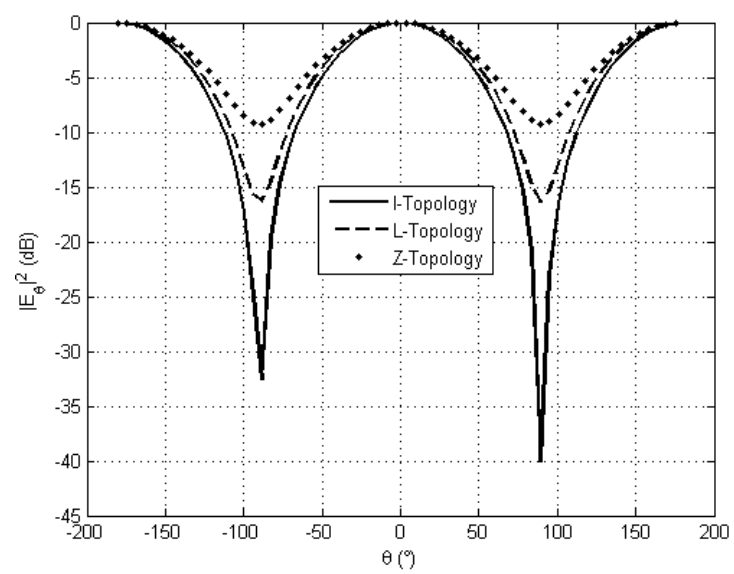

Figure 6: 2-D radiation pattern of microstrip dipole antenna Figure 6 shows 2-D radiation pattern of mono-plane microstrip dipole antenna in its variant topologies in the Eplane for which $\phi=0^{\circ}$. We observed that shadow areas are not enough marked such as illustrated by the radiation patterns of printed mono-plane dipole antenna in $\mathrm{L}$ or $\mathrm{Z}$ topology. All of these observations contests to say that if we further complicating the antenna, we get a quasi-uniform radiation pattern and thus an antenna close enough of the perfect antenna. 


\section{International Journal of Science and Research (IJSR) ISSN (Online): 2319-7064}

Index Copernicus Value (2013): 6.14 | Impact Factor (2015): 6.391

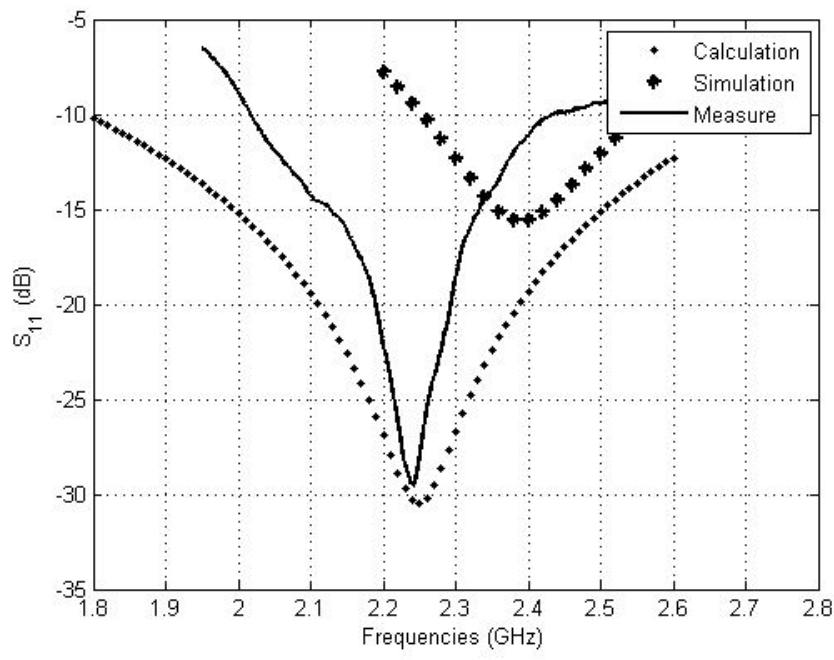

Figure 7: return less of microstrip dipole antenna in Itopology

Figure 6 illustrates the evolution of the $S_{11}$ parameter of the printed dipole antenna in I-topology. Calculation and measurement data show the shift of the resonant frequency with a bandwidth of about $40 \%$ whereas the simulated data indicate the resonant at the work frequency.

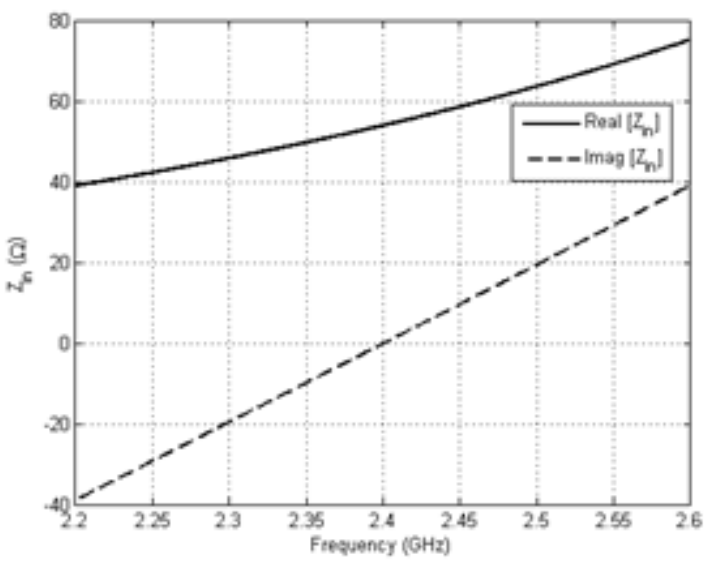

(a)

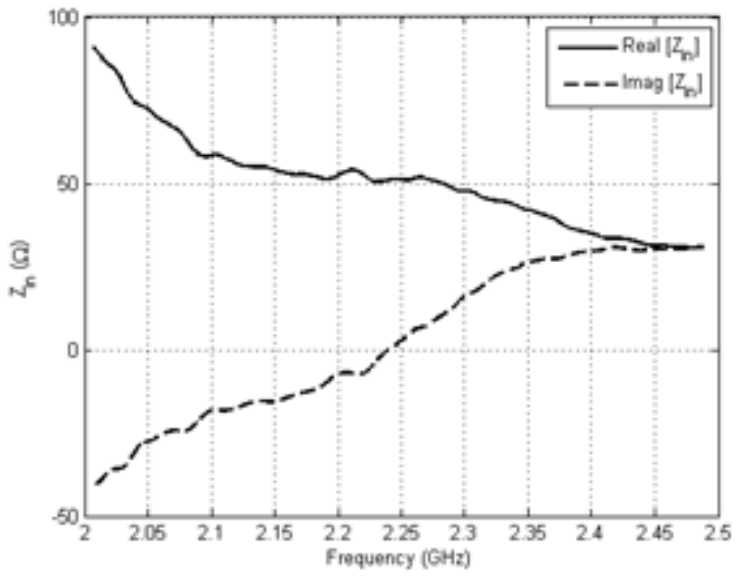

(b)

Figure 8: Input impedance of microstrip dipole antenna in Itopology

(a) Simulation (b) Measure

Figure 8 illustrates the simulated and measured data of the input impedance of mono-plane microstrip dipole antenna in
I-topology. Theoretically the evolution of the input resistance seems to follow a linear behavior with an input resistance of approximately $70 \Omega$ for a resonant frequency of about $2.42 \mathrm{GHz}$. While the measured data show that the input resistance decreases with a resonant resistance of the order of $50 \Omega$ for a resonant frequency turning around of $2.25 \mathrm{GHz}$.

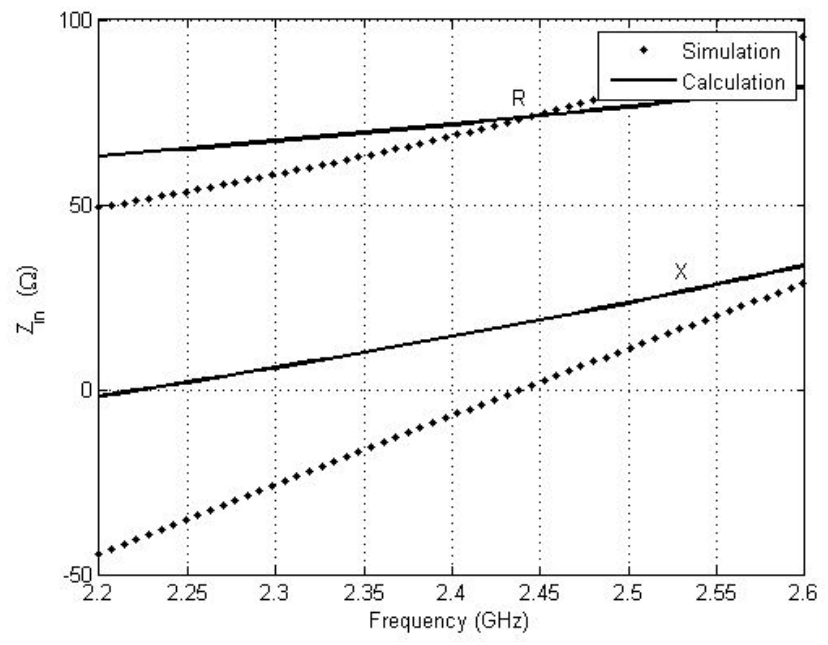

(a)

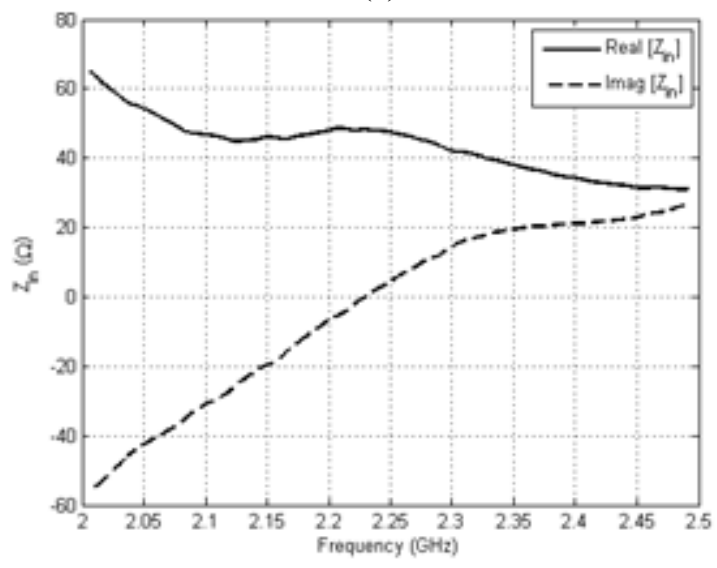

(b)

Figure 9: input impedance of microstrip dipole antenna in Ltopology
(a) Simulation
(b) Measure

As figure 8 , figure 9 illustrates the simulated and measured data of the input impedance of mono-plane microstrip dipole antenna in L-topology. Concerning the numerical simulation, input resistance has a linear behavior with an input resistance of approximately $50 \Omega$ for a resonant frequency of about 2.4GHz. While the measured data show that the input resistance decreases with a resonant resistance of the order of $50 \Omega$ for a resonant frequency turning around of $2.25 \mathrm{GHz}$.

An objective observation between the data of figure 8 and those of figure 9 allows to say that there is improvement in terms of input resistance that pass from $70 \Omega$ for the dipole antenna in I-topology to $50 \Omega$ for the dipole antenna in Ltopology even if the resonant frequency remain experimentally shifted from the working frequency. The fact of making bends on the radiation arms of the mono-plane printed dipole antenna in I-topology allows in reality to improve the impedance-matching and bandwidth.

\section{Volume 5 Issue 7, July 2016 www.ijsr.net}




\section{International Journal of Science and Research (IJSR) \\ ISSN (Online): 2319-7064}

Index Copernicus Value (2013): 6.14 | Impact Factor (2015): 6.391

\section{Conclusion}

We developed a design approach of new topologies of monoplane microstrip dipole antenna that approximates the perfect antenna. We then explored their potentialities in terms of radiation pattern and input impedance. Experimental and simulation investigations were conducted using transmission lines theory in travelling wave. These new radiating elements obtained by changing the profile of the mono-plane microstrip dipole antenna ensure bandwidth enhancement and impedance-matching. They are illustrated as the elements of the future because of their good performances, their low weight and their ease of design. Theoretically we found that their input impedance is close enough to a linear behavior while experimentally it is in perfect agreement with theoretical predictions.

\section{References}

[1] C. A. Balanis, Antenna theory: analysis and design, John Wiley \& Sons, 2012.

[2] Jiangniu $\mathrm{Wu}$, Zhiqin Zhao, Zaiping Nie and Qing-Huo Liu, "A Compact Printed Dipole Antenna for Wideband Wireless Applications," Progress In Electromagnetics Research C, (50) pp. 95-102, 2014.

[3] Prabir Banerjee and Tulshi Bezboruah, "Investigations on Modeling and Simulation of Printed Folded Dipole Antenna," International Journal of Hybrid Information Technology, (7), pp. 375-388, 2014.

[4] Nitali garg, Dr. Zarreen, "Design of Microstrip Dipole Antenna at Various Ground Plane," International Journal of Innovative Research in Sciences, Engineering and Technology, pp. 182-184, 2012.

[5] Nitali Garg and Zarreen Aijaz, "A Dual Band Microstrip Dipole Antenna for Wideband Application," International Journal of Computer Technology and Electronics Engineering, pp. 18-20, 2012.

[6] M. J. Jamaluddin \& al., "Microstrip Dipole Antenna Analysis with Different Width and Length at 2.4GHz," Asia-Pacific Conference on Applied Electromagnetics Proceedings Malaysia, pp. 41-44, 2005.

[7] Dong-Jin Kim \& al., "A Small Monopole Antenna with Novel Impedance Matching Structure," Proceedings of the $36^{\text {th }}$ European Microwave Conference, pp. 819-822, 2006.

[8] C. Laohapensaeng \& al., "Simplified integral equation for analysing the printed strip dipole antenna," IEE Proc.-Microw. Antennas Propag. Vol. 153, (3), pp. 301306, 2006.

[9] Constantinos VOTIS, Vasilis CHRISTOFILAKIS and Panos KOSTARAKIS, "Geometry Aspects and Experimental Results of a Printed Dipole Antenna," Int. J. Communications, Network and System Sciences, (3), pp. 204-207, 2010.

[10] John McVay, Ahmad Hoorfar and Nader Engheta, "Radiation Characteristics of Microstrip Dipole Antennas over a High-Impedance Metamaterial Surface made of Hilbert Inclusions,” IEEE, pp. 587-590, 2003.

\section{Author Profile}

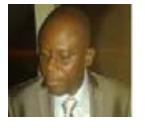

Dr Clément Mbinack is Senior Lecturer at University of Yaoundé I, Faculty of Sciences, Department of Physics. His research field covers inter alia smart antennas for WLAN application, electromagnetic modeling, microstrip antennas, Genetic Algorithms optimization and Automatic Systems. He was worked at "Groupe MOSE" of ISAE-SUPAERO from France-Toulouse as trainee in the mobility program of the Agence Universitaire de la Francophonie.

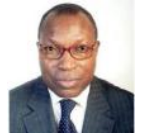

Professor Emmanuel Tonye is the manager of the Laboratory of Electronic and Signal Processing at the Engineering Advanced School of University of Yaounde I. His work researches cover multiple fields such as Telecommunications, Remote Sensing, Educational Engineering. He now occupies the functions of Vice-Rector in Charge of Research, Cooperation and Relations with Business World at University of Yaoundé I. 